\title{
Das Alphabet der Natur und das Alphabet der Kultur im 18. Jahrhundert
}

\author{
Botanik, Diplomatik, Linguistik \\ und Ethnographie nach Carl von \\ Linné, Johann Christoph Gatterer \\ und Christian Wilhelm Büttner
}

\author{
Martin Gierl
}

The Alphabet of Nature and the Alphabet of Culture in the Eighteenth Century. Botany, Diplomatics, and Ethno-Linguistics according to Carl von Linné, Johann Christoph Gatterer, and Christian Wilhelm Büttner

In the middle of the eighteenth century, Carl von Linné, Johann Christoph Gatterer, and Christian Wilhelm Büttner attempted to realize the old idea of deciphering the alphabet of the world, which Francis Bacon had raised as a general postulate of science. This article describes these attempts and their interrelations. Linné used the model of the alphabet to classify plants according to the characters of this fruiting body. Gatterer, one of the leading German historians during the Enlightenment, adopted the botanical method of classification by genus and species to classify the history of scripts. He used the forms of the alphabetic characters to measure the age of manuscripts and to map the process of history as a genealogy of culture. Gatterer collaborated closely with Büttner, the first Göttingen professor of natural history. Büttner constructed a general alphabet of languages which connected the phonetics of language with the historically known alphabets. Early on, diplomatics and ethnography combined the natural order of natural history and the cultural order of the alphabet with the attempt to register development and to document development by the evolution of forms. Based on the shared model of the alphabet and on the common necessity to classify their empirical material, natural history and the description of culture were related attempts in the middle of the eighteenth century to comprehend the alphabetically organized nature and a naturally ordered culture.

Keywords: alphabets, organization and categorization of knowledge, history as science, book of nature, diplomatics as botany 
In der Mitte des 18. Jahrhunderts konstatierte man die „,natürliche Ordnung" der Welt, so ein Schlagwort des aufgeklärten Wissenskabinetts, nicht mehr nur, sondern rekonstruierte sie am empirischen Material. Die für die moderne Wissenschaft zentrale Verbindung von Naturphilosophie und Historia naturalis hat sich hier vollzogen und die sich entwickelnden Kulturwissenschaften standen nicht abseits dabei. Für Johann Christoph Gatterer (17271799), der in Göttingen Geschichtsprofessor war und als zentraler deutscher Aufklärungshistoriker gilt, für Christian Wilhelm Büttner (1716-1801) als Linguisten, Ethnologen und Naturhistoriker sowie für Carl von Linné (1707-1778) waren die gottgeschaffene Natur und die von Gott gelenkte Kultur ein zusammengehöriger, ebenso kultureller wie natürlicher Prozess. Alle drei haben das Alphabet der Welt an den jeweils empirisch gegebenen Fachgegenständen auszubuchstabieren versucht, und sie haben dies in wechselseitiger Abhängigkeit betrieben. Diesen Verbindungslinien werde ich hier in den wesentlichen Zügen nachgehen und an den Alphabeten der Pflanzen, der Schrift- und der Sprachgeschichte, die bei Linné, Gatterer und Büttner herausgekommen sind, zeigen, wie Botanik, Diplomatik, Linguistik und Ethnologie, wie Historia naturalis und civilis, wie Natur- und Kulturbearbeitung zwischen 1750 und 1770 zusammengehörten. ${ }^{1}$

Der kultur- und wissenschaftsgeschichtliche Vorlauf dieser Episode in der Rekonstruktion des Alphabets der Natur und des Alphabets der Kultur ist immens. Am Anfang war das Wort, heißt es im Johannes-Evangelium, und die Gelehrten hofften, lesen zu können, wie es in den Dingen eingeschrieben ist. Nicht erst seit der Renaissance, aber nun als mehr und mehr systematisches Unternehmen hatte man von der magischen Kraft der Zeichen kabbalistischer Gelehrsamkeit bis hin zu den praktischen Universalsprachensystemen des späten 17. Jahrhunderts das Alphabet der Welt zu deuten und nachzuahmen versucht (Drucker 1995). Das „Alphabet“ war Metapher, Metonymie und Modell zugleich. Welches Bild lag näher, war überhaupt möglich, wollte man über die Analyse und das Verstehen einer fremden Natur reden, mit und in der sich Gott ausgesprochen hatte, die jedenfalls in Maßen Sinn besaß? Anfang des 17. Jahrhunderts erklärte 
Francis Bacon das „Alphabet der Natur“ zum Zielobjekt und zur methodischen Folie des Novum organon einer neuen Wissenschaft, die die Natur zu weiterem Gebrauch entschlüsseln sollte (Pernkopf 2004: 81). Nicht das Bild allein, sondern die Verbindung von Bild und methodischem Programm ist das Interessante dabei wie das Bild in der Wissenschaftspraxis umgesetzt wurde und von da gemäß dem jeweiligen Gegenstandsfeld Wissen organisierte. Gatterer schrieb:

Freilich ist, wie alles in der Welt, so auch die menschliche Schrift, vielen, sehr merklichen, Veränderungen unterworfen. Es ist mit den verschiedenen Arten der einzelnen Buchstaben, wie mit den Menschen selbst. Nicht ein ganzes Volk entsteht auf einmal und plötzlich, wie aus der Erde hervorwachsende Schwämme: auch stirbt ein ganzes Volk in allen seinen Individuen nicht auf einmal aus. Eben so ändert sich nicht auf einmal das ganze Alphabet einer Schriftgattung, sondern nach und nach, und einzeln, die eine Figur früher, die andere später. ([Gatterer] 1786: 1946)

Auch Völker erscheinen hier wie Schriften als Gattungen, die durch die je spezifische Form ihres Alphabets definiert werden - die Rede von Abstammung und Entwicklung einzelner Elemente erschien in der Diplomatik früh. Aber zunächst zur Botanik und zu Linné.

\section{Linnés Fruktationsbuchstaben}

Wir erkennen keine Autorität in der Botanik an, außer die persönliche Beobachtung. [...] Wahrlich, es gibt hier mehr Teile, mehr Buchstaben, als bei den Buchstaben der Sprachen oder den Alphabeten. Diese Merkmale sind uns alle Buchstaben der Pflanzen, aus deren Lesen wir die Kennzeichen der Pflanzen lernen. Diese hat der Schöpfer eingeprägt. Und diese zu lesen, werden wir uns bemühen. (Linné 1737: Ratio operis $\$ 11$, zit. nach MüllerWille 1999: 212)

Diese 26 Buchstaben der Pflanze fand Linné vor:

Wir haben bey dem Kelche (1) die Hülle, (2) die Scheide, (3) die Blumendecke, (4) das Kätzgen, (5) das Bälglein, (6) die Haube.

an der Krone (7) die Röhre oder Nägel, (8) die Mündung, (9) das Honigbehältniß.

an den Staubfäden: (10) die Träger, (11) die Staubbeutel.

an den Stempfel: (12) den Fruchtknoten, (13) den Griffel, (14) die Narbe. an der Frucht: (15) die Kapsel, (16) die Schote, (17) die Hülse, (18) die Nuß, (19) die Steinfrucht, (20) die Beere, (21) die Kernfrucht, an den (22) Saamen, (23) dessen Krone.

den Boden: (24) der Blume, (25) der Staubfäden, (26) des Fruchtknotens. (Linné 1737: Ratio operis $\$ 11$ zit. nach Linné 1775, Einleitung)

So entwarf Linné das Programm der Botanik in der "Ratio operis“ der Genera plantarum von 1737. War das 18. Jahrhundert das Zeitalter, das Klassifikation mit Empirie verband, so steht Carl von Linnés Klassifikationssystem paradigmatisch dafür. Er hatte 
nach einer zweifachen, systematisch artifiziellen Unterscheidung in Klassen und Ordnungen die Tier- und Pflanzenwelt in natürlich empirische Gattungen, Arten und schließlich Varietäten gegliedert. $^{2}$ Für die fünffache Staffelungstiefe nutzte Linné eine Reihe von Analogien. Auch beim Militär werde fünffach unterteilt von der Legion über die Kohorte hinab zum Soldaten. Die Kategorisierungslogik der Philosophie hierarchisiere nach oberster, mittlerer, zugehörender Gattung, Art und Individuum, und in der Geographie unterscheide man nach Reich, Provinz, Gebiet, Gemeinde und Flecken (Linné 1751: 98). Die Symmetrie scheint an dieser Stelle nicht nur die Adäquatheit und Praktikabilität des Ordnungssystems, sondern mit der Harmonie von Natur und Kultur auch das Naturgemäße der Unterteilung zu belegen. War die Welt gut, wahr und schön zugleich, so war sie im Denken der Zeit mechanisch, ästhetisch und als Gesamtökonomie nützlich organisiert.

Unter der ÖKONOMIE DER NATUR verstehen wir die äußerst weise Anordnung, die der Schöpfer bei den Naturdingen getroffen hat, nach der diese zu gemeinsamen Zwecken und wechselseitigen Nutzen geeignet sind. (Linné 1749/1787: 2 f., Hervorh. im Original, zit. nach Müller-Wille 1999: 272 f. $)^{3}$

Alles sei in Serien und gegenseitiger Verknüpfung geordnet. Der Begriff der Serie ist noch heute mit der Genealogie verbunden und über sie mit Verwandtschaft, Familie, Herrschaft und Staat. $^{4}$

Wie hingen Natur, Beobachtung, das Lesen, Beschreiben und das Alphabet der Pflanzen für Linné zusammen? Grundsätzlich galt auch für Linné wie für andere Botaniker: „Die Beschreibung ist die natürliche Kennzeichnung der ganzen Pflanze, welche alle äußeren Teile derselben beschreibt." (Linné 1751: 256, vgl. 257, Übersetzung Müller-Wille 1999: 223) Ein Aspekt kam bei Linné entscheidend hinzu: Gott habe die Individuen beauftragt, im Netz der Serien Fortpflanzungsarbeit zu leisten. Fruchtkörper und Fruchtbildung waren das, was es zu lesen galt. Man müsse die charakteristischen Merkmale einer Pflanze aus der Zahl, Gestalt, Proportion und Lage aller Teile der Fruchtbildung beziehen, heißt es in der Philosophia botanica. Multipliziert man die 26 „Buchstaben“ der Pflanze mit Zahl, Gestalt, Proportion und Lage, ergibt das nach Linné die „Nota characteristica“ der Pflanzen und damit die Gattungen (Linné 1737: Ratio operis $\$$ 19, 1751: 117). Die Elemente des Fruchtkörpers bilden dabei die charakteristisch geformten Zeichen der Fruchtbildung, die als Wesen der Pflanzen 
im Mittelpunkt der Linné'schen Botanik steht (Müller-Wille 1999: 223-264). Denn die „Fruchtbildung ist ein temporärer, der Zeugung gewidmeter Teil der Vegetation, die alte abschließend, die neue beginnend" (Linné 1751: 52). ${ }^{5}$ Linné ging es darum, mit dem Fruchtkörper das Wesen der Pflanzen in Form natürlich vorliegender Zeichen zu erfassen.

Oft haben andere Geschmack, Geruch, Farbe, Grösse ohne Maasstab zu Hülfe genommen. Diese wird man niemals von mir angeführt finden; sondern nur jene vier gewisse und beständige mechanische Grundsätze: die Anzahl, Gestalt, Lage und Verhältniß. Diese vier Eigenschaften, und jene 26. Buchstaben (\$. 11.) unterscheiden die Gattungen auf das gewisseste von einander. (Linné 1737: Ratio operis $\mathbb{\$} 19$, zit. nach Linné 1775 , Einleitung)

Linnés „natürliche Merkmale“ der Pflanzen bildeten ein botanisches Schriftsystem, das analog zur universalen Beschreibungspotenz der Buchstabenschrift zwei für die Botanik wesentliche Vorteile aufwies. Der Nutzen und die Vorzüge der natürlichen Merkmale sind:

1. Diese Merkmale können auf alle Methoden, die man schon hat, und noch erwarten kann, angewendet werden, wenn nur das System auf den festen Grunde der Blumentheile errichtet ist. [...]

2. Und wenn noch tausend neue Gattungen entdeckt würden, so würde man deswegen doch nicht ein einziges Kennzeichen der nächsten natürlichen Gattung hinzuthun, oder hinwegzunehmen genöthiget seyn. (Linné 1737: Ratio operis $\mathbb{\$} 18$, zit. nach Linné 1775 , Einleitung).

Ein weiterer, entscheidender Vorteil für die Klassifikation der Pflanzen kommt nach Linné durch die „natürliche“ Übersetzung der Pflanzenteile in das botanische Alphabet hinzu. Die Pflanzen beschreiben und bezeichnen sich quasi selbst: Die natürlichen Merkmale „drücken immer den nemlichen Begriff aus, wenn auch der Nahme tausendmal geändert wird“ (ebd.). Linné teilte die so „natürlich“ beschriebenen Gattungen in der obersten Taxa des Pflanzensystems Klassen zu. Charakterisierte die Fruchtbildung die Gattung, so charakterisierte das Sexualsystem die Klasse: 24 Klassen entstanden aus dem Vorhandensein, der Anzahl und der Form der Staubgefäße und Fruchtstempel. Das Alphabet der Fruchtkörper mündete derart in das übergeordnete Alphabet der Fortpflanzung, dessen „Literae“ Linnés Kupferstecher Georg Dionysius Ehret ursprünglich nicht durchnummeriert, sondern mit den 24 Buchstaben des lateinischen Alphabets gekennzeichnet hatte (Abb. 1; vgl. Feuerstein-Herz 2007: 104). 
CAROLI LINNAIT CLASSESSS.IITERAE.
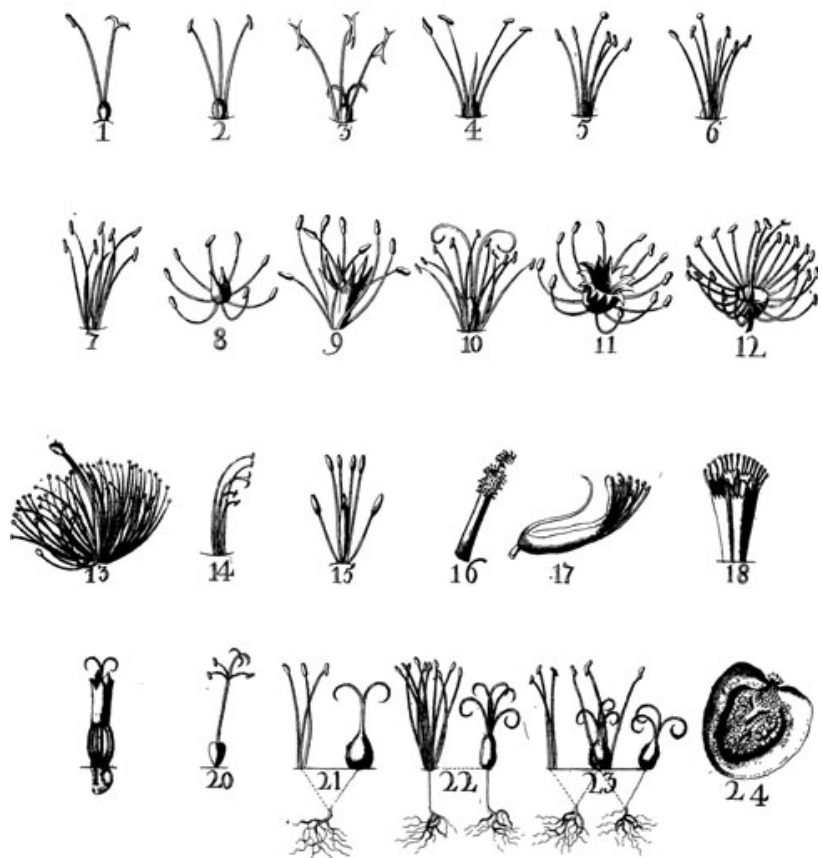

Classes S. Literae (Linné 1737).

\section{Johann Christoph Gatterer und der Linnaeismus graphicus}

Gatterer gilt nicht nur als führender Aufklärungshistoriker, sondern auch als derjenige, der die Hilfswissenschaften - insbesondere die Genealogie, aber auch die Diplomatik - in Deutschland zur Wissenschaft machte. ${ }^{6}$ Urkunden sind, definierte er, das Ergebnis derjenigen feierlichen und verbindlichen Prozedere, die Rechte und Tatsachen setzen. In ihnen ist der Rechtsbildungsprozess zusammengefasst (Gatterer 1798: 1). Sie entsprechen somit einer Art Fruchtkörper der Geschichte, in denen sich die „merk“-, das heißt die gedächtniswürdigen (Rechts-)Ereignisse der Geschichte konzentrieren. Ein direkter Weg führt von hier zu Linné, und tatsächlich hat Gatterer das Kernstück seiner Diplomatik Linnaeismus graphicus genannt. ${ }^{7}$ Die Historiker der Frühen Neuzeit definierten Historie übereinstimmend als Überlieferung der „merkwürdigen“ Ereignisse (Blanke/Fleischer 1990). Ihr Zusammenhang, ihre Serie, konstituierte Geschichte als Genealogie. Als Rechts- und Verfassungsgeschichte wenigstens lag sie in den 
Urkunden vor, mit denen jeweils ein Stück alte Geschichte endet und ein Stück neuer Geschichte begann. Die Urkunden empirisch - „historisch“ ist der zeitgenössische Begriff für empirische Fakten - zu verifizieren, bedeutete, Geschichte zu objektivieren.

Gatterers Versuch, Diplomatik empirisch präzise werden zu lassen, war nicht neu, sondern beruhte nicht unwesentlich auf Streitigkeiten zwischen den Benediktinern und Jesuiten. ${ }^{8}$ Seit Mitte des 17. Jahrhunderts hatten Antwerpener Jesuiten, die Bollandisten, das Großprojekt der Acta Sanctorum betrieben. Einer von ihnen, Daniel Papebroch, erklärte in den 1670er Jahren die meisten Merowinger Urkunden der Benediktiner für falsch. Daraufhin stellte deren Archivar Jean Mabillon von Saint-Maur, einer der sogenannten Mauriner, in einem ausgesprochenen Grundlagenwerk der Diplomatik die Möglichkeiten der Urkundenverifizierung detailliert dar. Die Debatte legte sich nicht. Im Gegenteil, der Jesuit Jean Hardouin ging soweit, nahezu die gesamte ältere Textüberlieferung - auch die der Kirchenväter - als Fälschungen zu betrachten. Die Mauriner Charles Toustain und René Tassin konterten mit ihrem sechsbändigen Werk Nouveau Traité de diplomatique (1750-1765), das die Gegner zum Schweigen bringen sollte. ${ }^{9}$ Dazu dokumentierten sie die lateinische Schrift in ihrer Entwicklung. Man sei dabei der Naturlehre gefolgt, denn schließlich zeige sie die Weisheit des Schöpfers und habe Tiere und Pflanzen in eine vernünftige Ordnung gebracht. Am Ende standen alphabetische Tafeln mit mehr als 30.000 Buchstaben, die den Autoren zufolge aus 300.000 bis 400.000 Buchstaben ausgewählt worden waren ([Adelung] 1759-1769, Bd. 3: Vorrede der Verfasser). Allein der zweite Band weist 40 derartige Tableaus auf. Gatterer hielt das Werk für das wichtigste Buch der vergangenen 50 Jahre.

Von den Tabellen 22 und 23 war er begeistert (Gatterer 1799: 5). Tabelle 22 zeigt die Entwicklung der lateinischen Kursive, also der Schreibschrift, historisch-geographisch nach Spalten geordnet in ihrer römischen, lombardischen, westgotischen bis karolingischen und kapetingischen Variante. Tabelle 23 baut darauf auf und präsentiert die Buchstabenformentwicklung des 4.-17. Jahrhunderts für Italien, Frankreich, Deutschland, Großbritannien und Spanien (Abb. 2). Eine Tafel enthält bis zu 5.000 Zeichen. Die Einzelbuchstaben in den Spalten sind jeweils römisch nach Jahrhunderten und dann weiter arabisch nach Formtypen unterschieden. Auf diese Weise sollte die Buchstabenformentwicklung nachvollziehbar sein, und es lassen sich - gute Augen vorausgesetzt - 


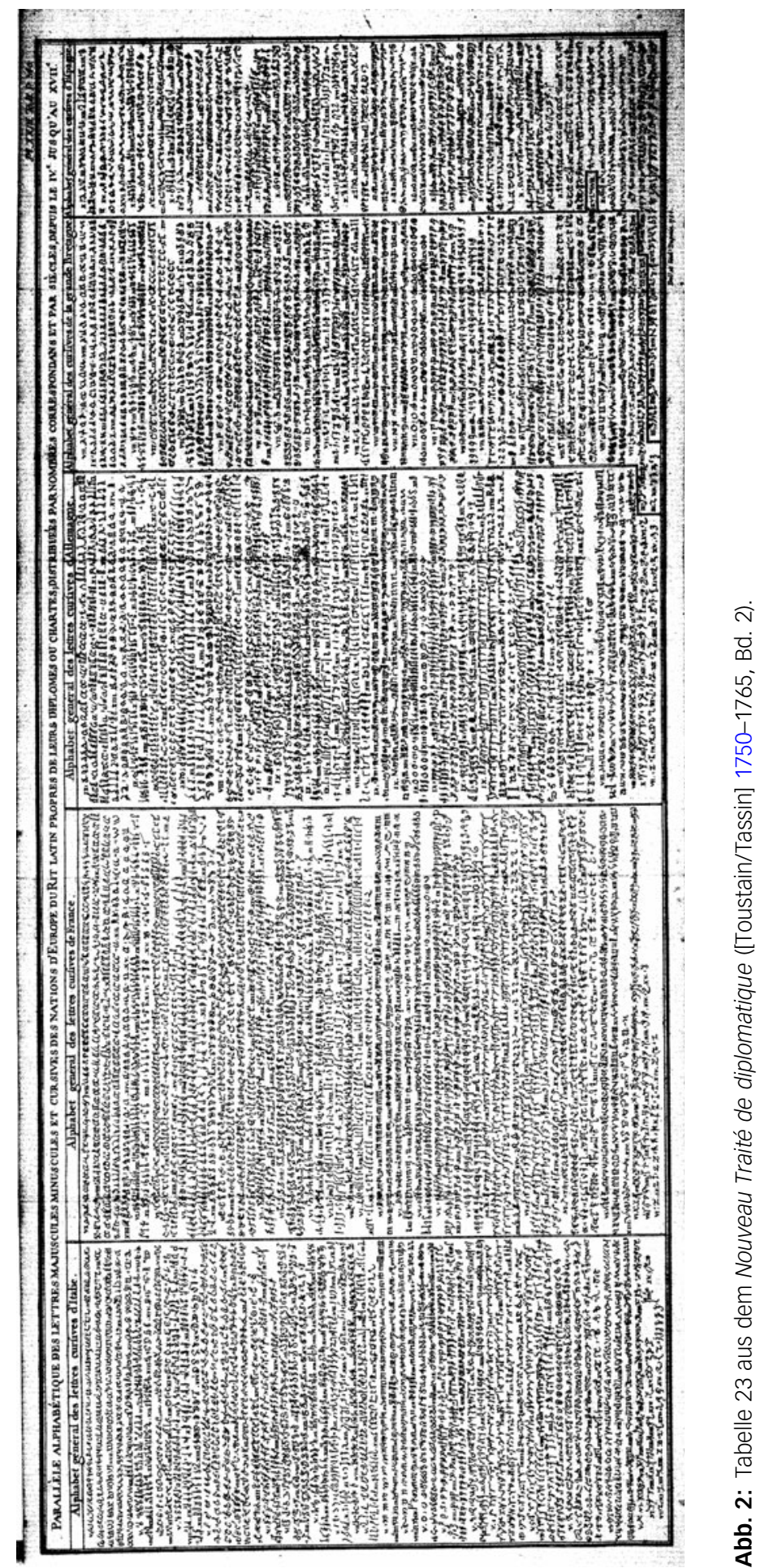




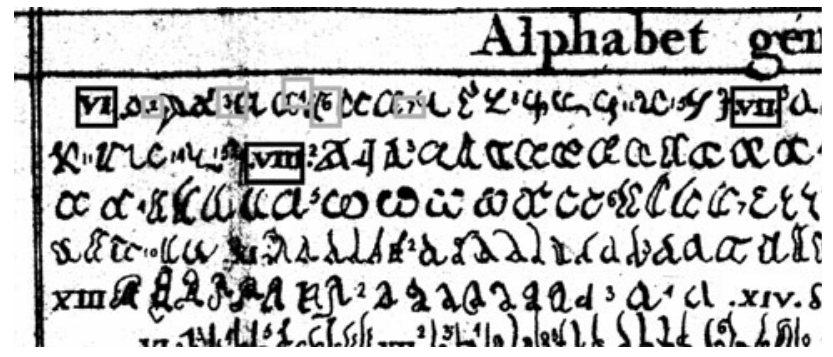

Abb. 3: Ausschnitt aus Tabelle 23 mit exemplarisch hervorgehobenen Jahrhundertangaben und arabischer Schrifttypennummerierung ([Toustain/ Tassin] 1750-1765, Bd. 2).

die Buchstaben nach ihren Formen den einzelnen Jahrhunderten sowie den Nationalschriften zuordnen. Darüber hinaus ist es möglich, qua Kennzeichnung mit arabischen beziehungsweise lateinischen Ziffern das Auftreten der einzelnen Formtypen zeitlich nach Jahrhunderten abzulesen (Abb. 3). Die Entwicklung der lateinischen Schrift lässt sich also zeitlich, geographisch und nach Formtypen der Schrift verfolgen ([Toustain/Tassin] 1750-1765, Bd. 2: 336-343). Man habe die Buchstaben so in die Tafeln gesetzt, um „den Faden der Abstammung der Figuren, die auseinander entstehen, nicht zu zerreissen und um die Stufen merklich $\mathrm{zu}$ machen, nach welchen die Buchstaben eines Theils volkomner geworden sind“ ([Adelung] 1759-1769, Bd. 3: 5).

Was Gatterer an den Tafeln faszinierte, war weniger die Formentwicklung selbst als die historisch nationale, geographische und chronologische Zuordnung von Schrift. Mit seinem Linnaeismus graphicus aus den 1760er Jahren versuchte er sie zu verallgemeinern. Es ging ihm nicht mehr nur um die lateinische Schrift als solche, sondern um die Klassen, Ordnungen, Serien, Gattungen und Arten aller Schriften, weltweit (Gatterer 1765: 81143, 1798: 39-53, 1806: 34-45).

Toustain und Tassin hatten Schrift in Stein- und Metallschriften (Inschriften), Manuskripte und schließlich Akten- und Urkunden unterteilt: „Ce sont comme les trois regnes de la nature.“ ([Toustain/Tassin] 1750-1765, Bd. 2: 6) Die drei Reiche der Schrift gliederten sie dann auf 1.000 Seiten nacheinander in Haupt- und Unterabteilungen, Gattungen und Arten entsprechend, und dokumentierten diese jeweils mit Beispielen von Inschriften, Münzen, Manuskripten und Urkunden in den Tafeln. Gatterer machte daraus ein „Regnum artificiale“ („Künstlerschrift", „Inschriften“), ein „Regnum librarium“ („Bücherschrift“, „Handschriften“) und ein „Regnum notariale“ („Kanzleyschriften“). ${ }^{10}$ Er unterteilte die Schriftreiche in die Klassen der Bilder-, Silben- und Buchstabenschrift, die Bilderschrift wiederum in die Ordnungen der „Idiopragmatische[n] 
oder Mexikanische[n] Schrift“ (Sachen als Bilder), der „Hieroglyphischen oder Aegyptischen Schrift" (Bildsymbole der Sachen) und die "Charakteren-Schrift, oder Chinesische-Schrift" (Logogramme; Sachen durch abstrakte Zeichen). Mit der Silben- und der Buchstabenschrift verfuhr er analog (Gatterer 1798: 39-53, vgl. 1806: 34-45; Hervorh. im Original).

Die Klassen und Ordnungen gaben nicht nur der Schrift an sich eine Taxonomie. Gatterer ordnete die Schriften zudem flächendeckend nach ethnographischen und geographischen Kriterien. Die Schrift fungierte somit über den klassifizierbaren Entwicklungsstatus als Marker für die kulturelle Entwicklung der Welt. Darüber hinaus plante Gatterer die Schriften in historische Serien zu unterteilen. Für die lateinische Schrift hat er dies, dem Nouveau Traité folgend, durchgeführt und sie nach drei Serien unterschieden: Schriften römischen Ursprungs, frühmittelalterliche und spätmittelalterlich frühneuzeitliche Nationalschriften. Kultur ist hier einem Entwicklungsschema mit Beginn-Ausbildung-Reife unterworfen und zugleich national verortet. Das dynamische Moment dabei wird noch deutlicher, geht man die Beschreibungen der historischen Serien durch, die Gatterer in der „Kurzfassung der Diplomatik“ gegeben hat. Hier wurden die „dominantium [...] scripturarum Latinarum mutationes“ (Gatterer 1806: 42) dem Nouveau Traité folgend in ihrer chronologischen Abfolge und wesentlichen Charakteristik aufgeführt und die historischen Serien nach Entstehung, Verbreitung und Dauer klassifiziert. Zum Beispiel:

Series I. Scriptura Romana vetus.

1) Orta est ex Etrusca $[\ldots]$

2) Dominata est in toto imperio Romano [...]

3) Duravit usque ad migrationes gentium sec. V. et VI. (Gatterer 1806: 46)

Schrift wird also nicht nur in der historischen Grobschichtung, sondern nach der historischen Ausdehnung und Ausbreitung erfasst.

Voller Bewunderung für die Linnésche Methode und in der festen Überzeugung, dass sich Natur und Kultur glichen, habe er den Linnaeismus graphicus entwickelt, aber auch, weil das Vorgehen in der Diplomatik etwas leiste, was es in der Naturhistorie nicht leiste: Es bestimme das Alter des diplomatischen Materials. So begründete Gatterer seinen Versuch, Schriftgeschichte Linné folgend $\mathrm{zu}$ betreiben und dabei mit der Verbindung von Schriftentwicklung und Diplomatik Kultur, Geschichte und Nationenbildungsprozess zu verknüpfen (Gatterer 1798: 37 f.). Tatsächlich war die mangelnde Verzeitlichung ein Kernproblem des Über- 
gangs der klassifikatorischen Naturgeschichte des 18. Jahrhunderts zu den Evolutionsideen des 19. Jahrhunderts (Lefèvre 1984: 216).

Mitte des 18. Jahrhunderts wurde in der Botanik, wie durch Linné, nach zwei artifiziellen oberen Taxa, den Klassen und Ordnungen, die das Material nach dichotomischen Einteilungsprinzipien formal gliederten, und zwei unteren Taxa klassifiziert, den Gattungen und Arten, die das Material nach empirischen Gegebenheiten verzeichneten (Lefèvre 1984: 187-259, 2003: 1 f., Mayr 1982: 158, 190). Dem ist auch Gatterer gefolgt. Analog zu Linnés Fruchtkörperelementen und ihrer nach Zahl, Gestalt, Proportion und Lage vierfachen äußeren Typik nahm er die Buchstaben des Alphabets und klassifizierte den Schriftkörper nach der Buchstabentypik der Kapitale und Unziale - das sind die beiden Majuskelschriftarten -, der Minuskel und Kursive (Gatterer 1765: 93 f.). Eine Gattung entstand dann danach, ob eine kursive oder nichtkursive Schrift nur Kapitale oder Kapitale und Unziale oder Kapitale, Unziale und Minuskeln und so weiter enthielt. Für Codices definierte Gatterer 24 Schriftgattungen: reine Kapitale, Unziale, Minuskel, Kursive und die Mischformen davon. Für die vorherrschende Kapitale notierte er:

I. Ennium: Reine Kapital. II. Plautinum: Kapital, vermischt mit UncialBuchstaben. III. Terentium: Kapital, vermischt mit Minuskel-Buchstaben. IV. Catonium: Kapital, vermischt mit Kursiv-Buchstaben. V. Vitruvium: Kapital, vermischt mit mehreren Arten von Buchstaben. VI. Caesareum: vermischt mit zusammengezogenen [...] Buchstaben. (Gatterer 1798: $60 \mathrm{f}$., Hervorh. im Original)

Für die Unziale, Minuskel und Kursive verfuhr er analog. Auf diese Weise ließ sich Schrift nicht nur bestimmen, sondern vermessen, und zwar durch zweierlei Möglichkeiten: Einmal, indem man die Buchstabenformen einer Schrift nach Kapitale, Unziale und Minuskel quantifizierte und so deren Verhältnis beim einzelnen Buchstaben wie für die gesamte Schrift bestimmte. Zum anderen konnte man Werkzeuge bauen, um das Alter der Schrift zu messen.

Als Beispiel für die Anwendung des Linnaeismus diplomaticus benutzte Gatterer die Tabula Peutingeriana (Gatterer 1799: 153 f.), die berühmte römische Straßenkarte, die 1753 von Franz Christoph von Schreyb herausgegeben worden war. Der versierte Schöpflin, ein im 18. Jahrhundert berühmter Straßburger Historiograph, hatte sie auf das 4. Jahrhundert datiert. Tatsächlich gehöre sie aber ins 13. Jahrhundert, so Gatterer. ${ }^{11}$

So formalistisch und willkürlich von der lateinischen Schrift abgeleitet Gatterers Gattungsbildungsverfahren erscheint, bildete 
es doch ein Raster, mit dem sich lateinische Schriften und Schriftentwicklung ausmessen und relational darstellen ließen. Gatterer zog zunächst aus der Tabula Peutingeriana die dort benutzten Schriften samt ihrer Buchstabenvarianzen und setzte sie in alphabetische Form. Drei Schriftformen unterschied er in der Peutinger Tafel. Im Alphabet der ersten fand er ausschließlich Kapitale sowie vier Unziale E, H, L, M. - Plautinum also. Bei der zweiten Peutinger-Schrift (Abb. 4) lagen die Verhältnisse komplizierter. Um sie zu bestimmen, zählte er Kapitale, Unziale und Minuskel der einzelnen Buchstaben aus und brachte sie in eine tabellarische Form: 44 Kapitale, 26 Unziale, 12 Minuskel die Kapitale ist vorherrschend, vermischt mit Unzial- und Minuskelbuchstaben: Vitruvium (Gatterer 1799: $167 \mathrm{f}$.). Die Tabelle liefert nicht nur den vorherrschenden Buchstabentyp der Schrift und infolge Gatterers Gattung, sondern für jeden einzelnen Buchstaben die numerischen Mischrelationen in optisch griffiger Spaltenform. Eine Art „Fingerabdruck“ der Schrift ist damit entstanden. Am Horizont des Verfahrens steht die systematische Aufnahme von Handschrift-Schriften und der Vergleich ihrer hochvariablen, charakteristischen „Fingerabdrücke“ in Form der quantifizierten Buchstabentypentabellen beziehungsweise der Schrifttyprelationen (Abb. 5). Derart klassifiziert ließe sich die Nähe einzelner Handschriften quantitativ beziffern, so Gatterer. Die Möglichkeit einer empirisch quantitativ fassbaren Schriftentwicklung geht weit über das hinaus, was der Gattungsschematismus zunächst zu versprechen scheint.

Um die Entstehungszeit von Manuskripten messbar zu machen, stellte Gatterer deren datierbare Elemente in „chronometrischen Tafeln“ zusammen: Schreibmaterial, Formatierung, nicht zuletzt die Buchstabenformen. Jedem einzelnen Element ordnete er nach Jahrhunderten die Zeitphase $\mathrm{zu}$, in der es in Gebrauch war (Gatterer 1799: 163 f.). Vergleiche man den Befund einer Urkunde mit den Elementen der Tafeln, dann ergebe sich rasch das

Abb. 4: Alphabet des zweiten Schrifttyps der Tabula Peutingeriana (Gatterer 1799, Tab. I).
Tab $\boldsymbol{X Y}$

PEVTINGERIANA TABVLA,

ad Menfionem deflomatecam pracarate

II. Nphabctum Lorpturae ficandale.
Ха авсг⿻コ一

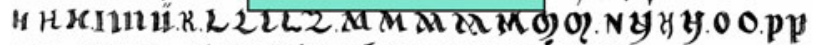
QqRk2.5ss.trt. 


\begin{tabular}{|c|c|c|c|}
\hline Litțerae & capitales & unciales & minutae \\
\hline a & - & 2 & I \\
\hline $\begin{array}{l}\text { b } \\
\text { c }\end{array}$ & I & - & 3 \\
\hline d & - & 3 & - \\
\hline e & 2 & 7 & 3 \\
\hline 5 & 5 & - & - \\
\hline h & 4 & - & - \\
\hline $\mathbf{i}$ & 6 & - & - \\
\hline k & $\mathbf{r}$ & - & 一 \\
\hline 1 & - & 5 & - \\
\hline $\mathbf{m}$ & 5 & 2 & 一 \\
\hline $\mathbf{n}$ & 4 & 一 & - \\
\hline o & 2 & - & 一 \\
\hline p & - & - & 2 \\
\hline $\mathbf{q}$ & $=$ & 2 & 一 \\
\hline $\mathbf{r}$ & 3 & - & - \\
\hline 8 & 4 & - & - \\
\hline $\mathbf{t}$ & 2 & - & $\mathbf{I}$ \\
\hline $\mathbf{u}$ & I & 一 & - \\
\hline $\mathbf{x}$ & 2 & - & - \\
\hline$y$ & 2 & - & - \\
\hline $\mathbf{z}$ & - & 2 & - \\
\hline Summa & 44 & 26 & 12 \\
\hline
\end{tabular}

Abb. 5: Quantitative Gattungsanalyse des zweiten Peutinger-Schrifttyps (Gatterer 1799: 170).

Jahrhundert, in dem alle Charakteristika der Urkunde noch oder schon gängig waren. Die Entstehungszeit der Urkunde werde so ablesbar, erklärte Gatterer. Neben chronometrischen Tafeln zur äußeren Form von handschriftlichen Texten, etwa dem Format, und Tafeln zu inneren Kriterien, etwa signifikanten Abkürzungen, hatte Gatterer chronometrische Tafeln nach Buchstabenformen im Sinn. Abbildung 6 zeigt die Entwicklung des Buchstaben A im Wesentlichen nach dem Nouveau Traité, von Gatterer exemplarisch in eine Datierungstafel umgesetzt.

Die Tabelle verzeichnet neben der Buchstabenform und der Zeitspanne des jeweiligen Buchstabengebrauchs den nationalen paläographischen Kontext sowie den Beleg. Der Rückgriff auf empirisches Material lässt es $\mathrm{zu}$, den einzelnen Buchstaben geschichtlich zu verorten, und nötigt zugleich, Nachprüfbarkeit zu offerieren.

In der für das 18. Jahrhundert typischen Verbindung von Wissenschaftlichkeit, Praktikabilität und Nutzen hatte Gatterer 


\section{$T a b . I V$.}

$\mathrm{s} \mathrm{pecimcn}$

Chronometrias fpecialis.

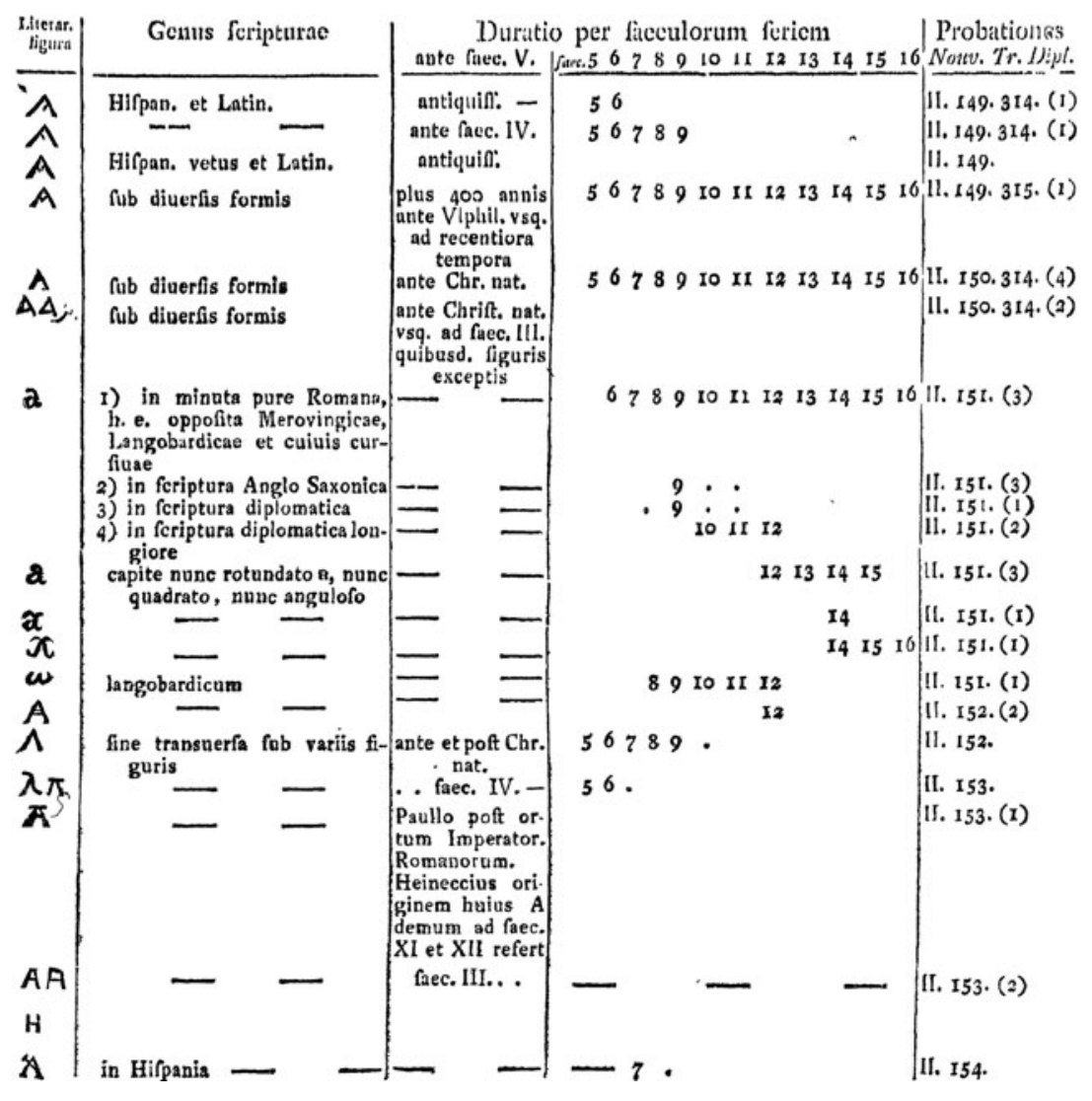

Abb. 6: Chronometri- mit dem Linnaeismus graphicus und seiner Verschränkung mit der sche Tafel zum Buchstaben A (Gatterer 1787, Tab. IV). Chronometrie an vorderster Stelle die instrumentelle Anwendbarkeit der Sache im Sinn. Die reale Kriterienvielfalt einer Schrift werde durch die Umsetzung empirisch gegebener diplomatischer Elemente zu chronometrischen Tafeln für das Auge der Wissenschaft wie für das der Laien und Schüler datierbar, weil die Tafeln Maßstäbe bieten, so Gatterer. Denn: „Wie [...] zu jeder Messung Maßstäbe nothwendig sind, so sind sie es auch bey dieser" ([Gatterer] 1786: 1948). Und „so könnte selbst ein Anfänger in der Diplomatik, wenn er nur gesunde Augen und gehörige Aufmerksamkeit mit zur Arbeit brächte, in einem Paar Stunden das Alter eines jeden gegebenen Codex mit diplomatischer Gewißheit bestimmen“ (ebd.: 1950). Bislang habe man mühsam Urkunden mit 
den Schriftproben in diplomatischen Handbüchern verglichen. Was Gatterer in Bezug auf die Chronometrie und Diplomatik anführte, folgte argumentativ exakt dem, was Linné über den Nutzen seiner Klassifikationsmethode und der „Fruktationsbuchstaben" geschrieben hatte:

Laß zwei Studierende, der eine ein Systematicus, der andere ein Empiricus, mit einer ausgezeichneten botanischen Bibliothek in einen Garten voller ausländischer und unbekannter Pflanzen kommen: während der erstere durch Ablesung der Fruktationsbuchstaben die Pflanzen leicht in Klasse, Ordnung und Gattung aufstellt und dann nur einige Arten zu unterscheiden hat, so ist der letztere gezwungen, alle Bücher durchzublättern, alle Beschreibungen durchzulesen und mit vieler Mühe alle Abbildungen zu prüfen, eine Sicherheit, welche Pflanze er vor sich hat, erhält er aber doch nicht, wenn nicht durch Zufall. (Linné 1737: Ratio operis $₫ 29$, zit. nach Lindman 1909: 53) 12 $^{12}$

Bis zu diesem Punkt hatte Gatterer seinen Linnaeismus graphicus ausgearbeitet. Die Homologie mit der Botanik ist jedoch, wenigstens der durchgängig spürbaren Intention nach, nicht auf die Schriftenklassifikation beschränkt. Auch jenseits dessen, was Gatterer „Schrift-Linnäism“ (Gatterer 1798: 37) nannte, das heißt im praktischen Beschreiben der Urkunden und der Urkundenanalytik, war seine Diplomatik an die botanische Praxis angelehnt. Gatterer teilte die Diplomatik in die Schriftenkunde (die "Graphica"), die Zeichenkunde (die "Semiotica“) und die von ihm wenig detaillierte Formelkunde ein, die mit Eingangs-, Schluss- und Textformeln das enthalten sollte, was die Diplomatik heute als innere Merkmale der Urkunde erfasst (ebd., Vogtherr 2008: 63-74). Die Semiotica beschreibt mit den Chrismen (einem verzierten "C" als symbolische Anrufung Christi zu Beginn der Urkunde), Notariats-, Investiturund Kreuzzeichen, Monogrammen und Siegeln die Identifikationsund Beglaubigungszeichen der Urkunden und damit den Zeichen gewordenen, feierlichen formalen Akt. So wie Linné die Elemente der Fruchtbildung zum Gattungsalphabet der Botanik werden ließ und nach Anzahl, Gestalt, Größenverhältnis und Lage analysierte, untersuchte Gatterer nicht nur die Buchstaben, sondern auch die sechs Zeichengruppen der Semiotica jeweils nach ihren Gestaltungsdetails auf der Urkunde und teilte sie nach Gattungen und Arten ein. Nicht die Urkunden insgesamt als Klasse historischer Texte wurden aber analysiert, sondern deren einzelne Teile, die einzelnen Beglaubigungszeichen wie die Siegel oder Monogramme und auf diese Weise jedes wiederum für sich. Gatterer blieb in der Kategorisierung der Zeichenkunde damit sachadäquat formal. Einen Linnaeismus diplomaticus universalis konstruierte er nicht. Wenn Linné zur Artenbeschreibung die Beschreibung aller Teile der Pflanze nach Anzahl, Gestalt, Größenverhältnis und Lage soweit 
möglich in der Fachsprache forderte, entsprach dies jedoch sehr genau dem, was sich Gatterer unter einer Urkundenbeschreibung vorstellte und in der „Praktischen Diplomatik“ an einem Beispiel vorexerziert hatte:

I. Beschreibung nach der Schriftkunde.

1. Das Format ist länger, als breit [...]

2. Die Fraktur ist zitternd [...]

3. Linnäischer Charakter der Schrift: Kursiv, vermischt mit Minuskel [...]

4. Die Anzahl der Abbreviaturen [...]

II. Beschreibung nach der Zeichenkunde.

1. Chrismon der 3ten Klasse zu Anfang der Urkunde [...]

2. Monogramm, fehlt [...]

3. Rekognitionszeichen, fehlt $[\ldots]$

4. Siegel. Es ist 1) aufgedrückt, nicht angehängt [...] 2) ein Majestätssiegel von grossem Umfange [...] 3) Bilderschrift: der Kaiser in Majestätsgestalt [...] 4) Buchstabenschrift: im Kreise herumgeschrieben [...]. (Gatterer 1799: 12-14, vgl. Linné 1737, Müller-Wille 1999: 67-97)

Wie ist Gatterers Vorgehensweise im Vergleich zu Linnés Konzeption zu bewerten? Toustain, Tassin und Gatterer bezahlten, aus heutiger Sicht, das Primat des praktischen Nutzens einer Methode hoch. Mit Recht hat man Gatterer Inkonsistenzen vorgehalten (Rödel 1982: 229, 236). Waren die drei Reiche der Schrift wirklich voneinander getrennt? Alles jenseits der lateinischen Schrift blieb bloße formale Klassifikation. Im Übergang von der Schrift- zur Urkundenanalyse verlor sich der botanische Ansatz letztlich in traditionell diplomatischer Beschreibung. Bemerkenswerterweise hatte Gatterer an keiner Stelle das Verhältnis seiner Diplomatik zur Botanik im Detail diskutiert. Dagegen formulierte er seine mit dem botanischen Vorgehen verbundenen epistemologischen Erwartungen explizit: Das Verfahren stehe in der Diplomatik erst an seinem Beginn. Die bislang gezogenen Gattungsgrenzen seien unscharf, Ziel müsse es sein, ebenso klare und kohärente Gattungseinteilungen zu finden wie in der Botanik (Gatterer 1767: 184f.). Waren die gewonnenen Ergebnisse unscharf, dann weniger aufgrund der klassifikatorischen Vorentscheidungen, sondern gerade weil Gatterer wie Toustain und Tassin das historische Material empirisch, so wie es vorlag, eingeordnet haben wollte. Der Linnaeismus graphicus war kein vollständig ausgebildetes System. Es war vor allen Dingen ein sich an den zeitgenössischen naturhistorischen Klassifikationsverfahren orientierendes Projekt.

Trotz aller Mängel, den potenziellen Vorteil der naturhistorischen Methode in der Diplomatik hat Gatterer klar gesehen. Mit ihr ließ sich historische Entwicklung abbilden. Bei Linné blieben die Fruchtkörperbuchstaben sich selbst von Generation zu Generation gleich. Sie entsprachen hierin der traditionellen Vorstellung 
ihrer göttlichen Herkunft und erlaubten im Gleichbleiben erst Klassifikation „nach der Natur“. Das zentrale Problem der Diplomatik, in der es um die Authentizität von Urkunden ging, bestand dagegen in der Schriftentwicklung. Gelang ihre Klassifikation, war ein Kriterium gewonnen, mit dem der Geschichtsverlauf rekonstruiert werden konnte. Sicher ist das diplomatische Interesse, Entwicklung zu klassifizieren, im zeitgenössischen Umfeld der Historisierung der Naturgeschichte von der Geologie bis zur Embryologie zu sehen. Dennoch erscheint das Bemühen Toustains und Tassins bemerkenswert, Schriftengeschichte nicht mehr durch zeitzugeordnete Beispiele zu belegen, sondern durch akribisch abgebildete und klassifizierte Formentwicklungsreihen nachzuvollziehen. Ebenso interessant ist der Versuch Gatterers, über die Schriftentwicklung das historische Urkundenmaterial zu verifizieren und hierdurch den Rechtssetzungsprozess und somit die Nationenentfaltung als Kulturbildungsprozess nachzuvollziehen.

Sowohl Toustain, Tassin wie Gatterer hatten Schriftenentwicklung instrumentell für ihre Sachfragen genutzt. Auch wenn bei Gatterer von „Mutationen“ die Rede war (Gatterer 1806: 42): Keinem wäre es in den Sinn gekommen, die Formenentwicklung intern als Forschungsgegenstand in den Blick zu nehmen. Dies änderte sich ein stückweit bei Christian Wilhelm Büttner. Während bei Linné das Alphabet für die Methode stand und die Pflanzen den Untersuchungsgegenstand bildeten, war bei Gatterer das Alphabet in seiner äußerlichen Form der Untersuchungsgegenstand und die naturhistorische Empirie die Methode. Bei Büttner hingegen bildete das Alphabet den Untersuchungsgegenstand. Er fasste es als Schlüssel für den Zusammenhang von Sprache und Schrift und zugleich als methodisches Instrument auf, mit dem er Linguistik und Ethnographie verbinden wollte.

\section{Das Weltalphabet Christian Wilhelm Büttners}

Büttner, dessen Gelehrsamkeit so breitgefächert sei wie die Natur selbst, habe seit vielen Jahren die Arbeit unternommen, die Sprachen und Schriften aller Völker völlig zu erklären, schrieb Gatterer (1765: 40). Büttner hatte nicht nur mit Linné in Leiden ein Zimmer geteilt und die erste deutsche Professur für Naturgeschichte in Göttingen inne, sondern war auch Gründungsmitglied von Gatterers Institut der historischen Wissenschaften und firmierte immer wieder als Referenzpunkt bei dessen Linnaeismus graphicus. ${ }^{13}$ 
Tatsächlich stammte das Einteilungssystem der Alphabete in Klassen und Ordnungen von Büttner (Gatterer 1798: 39-52). Nicht nur Linné und die Benediktiner, sondern insbesondere die Zusammenarbeit mit Büttner haben Gatterers Linnaeismus als globales System erst möglich gemacht.

Büttner war in Göttingen nicht nur für Gatterer der Gewährsmann an der Schnittstelle von Kultur und Natur. Für den Orientalisten, Palästinaforscher und Akademiedirektor Johann David Michaelis ebnete er den Weg zur Naturgeschichte, und er inspirierte Heinrich Moritz Gottlieb Grellmann, dessen ,Zigeuner“ -Buch eine verheerende Langzeitwirkung entwickelte und der von Büttner die Idee der indischen Abstammung der Roma übernahm (Grellmann 1787: IX-XIV, Breger 1995, Hund 1996). Grellmann hatte den wissenschaftlichen Kern seines Buchs - die Wortschatzvergleichstafeln „Zigeunerisch“, „Hindustanisch“, „Teutsch“ - von Büttner übernommen (Grellmann 1787: 284). Nicht zuletzt war Büttner der Lehrer des Anthropologen Blumenbach, der die Idee der weißen kaukasischen Rasse entwickelte (Urban 1991, 2001, Plischke 1931, Schulz/Kuhn 2001).

Vor allem aber war Büttner Alphabetforscher. ${ }^{14}$ Seinen Fokus auf die kulturelle Natur und natürliche Kultur richtete er auf das Alphabet. Er wollte alle existierenden Alphabete diachron in ihrer Entwicklung darstellen, synchron vergleichen und daraus ein allgemeines phonetisches Weltalphabet gewinnen. Wie der Mensch vom Tier seien die Völker durch Sprache geschieden, nicht nur durch die Semantik der Wörter, sondern auch durch die Phonetik der Rede. In ihr spiegle sich die kulturelle wie natürliche Umwelt des Menschen. Klima, Landstrich, Lebensart hätten die Beschaffenheit des Redeapparats des Menschen geprägt (Büttner 1771: 3 f.). Für Büttner war das in den Alphabeten enthaltene Lautsystem der Rede eine Art Entwicklungscode der Sprache. Ließen sich die Alphabete der Völker in ihrem Bestand phonetischer Zeichen samt der Gemeinsamkeiten und Abweichungen rekonstruieren, so würden sich die Grenzen zwischen den Völkern festlegen und die gemeinsame historische Entwicklung der Ethnien nachvollziehen lassen (ebd., Büttner 1779).

Büttner hat wenig publiziert. $\mathrm{Zu}$ seiner Alphabetforschung erschienen nur ein Akademievortrag (1776) und zwei schmale Hefte mit dem Titel Vergleichungs-Tafeln der Schriftarten verschiedener vergangener und gegenwärtiger Zeiten, das erste 1771 und das zweite 1779 (Schlichtegroll u. a. 2004 [1802]: 194). Letzteres endet mitten im Satz. Doch auch so sind die Texte aufschlussreich genug. 
Büttners Programm war die Beschaffenheit - oder die „Wanderungen und Abartungen“, wie es sein Biograph ausdrückte (ebd.) der Völker und Sprachen. Es ging ihm dabei um die Sprachentwicklung in Bezug auf die Ethnographie sowie umgekehrt und insgesamt um kaum weniger als die Erfassung der Zivilisationsgeschichte.

[Zum Sprachverständnis] sind auch die besondere Beschaffenheit der Gliedmassen zur Rede einiger Völker, ihrer Nahrungsmittel und Lebensart, des Himmelsstriches, unter welchem sie leben, wie auch die Höhe und Tiefe der Lage des Ortes welchen sie bewohnen, in Erwegung zu ziehen, weil davon oftmals der Unterscheid der Aussprache abhänget. Auch die Verbindungen und Trennungen unter sich verändern ihre Sprachen, sie geben und nehmen ihnen Wörter, und nach dem Maase wie sie zu Veränderungen im Lebenswandel geneigt sind, ändern sich auch ihre Redensarten. (Büttner 1779: 3)

Die von Linné benutzte Idee der Ökonomie der Natur taucht hier auf. In vagen Umrissen sind Humboldts Biotope als Soziotope von Sprachgemeinschaften erkennbar, in denen nach Biologie, Geographie, Klima, Natur und Kultur die Umweltbedingungen Sprache hervorbringen und wandeln. Büttner wollte mit seinem Prästrukturalismus Sprache nicht nur lexikalisch und grammatikalisch klassifizieren, sondern auch Entwicklung fassbar machen. Sein Leitbegriff ist „Abstammung“ (ebd.: 6, 32). Zweierlei bringt er dabei zusammen: die Abstammung der Völker und die der Sprachen. In beiden Fällen ging es ihm um Entwicklung in Raum und Zeit. Büttner versuchte, die Ausbreitung von Sprachen und Völkern synchron geographisch und diachron genealogisch darzustellen. Der Text zu seinen Alphabettafeln ist der Versuch, eine vollständige Ethnographie der Weltbesiedlung - wenn auch kursorisch und noch wenig quellenkritisch - zu liefern (Büttner 1771: 5 f., 1779: 32 f.). Es ging Büttner nicht nur darum, zu zeigen, wie Schrift sich verbreitete, sondern auch wie Sprache und Schrift jeweils von einer zur anderen Sprachgemeinschaft gewandert und in ihre Lautartikulation übernommen und angenommen worden sind. ${ }^{15}$ Ein „Volk“ übernehme nicht einfach ein Alphabet. Es ändere je nach eigener Sprachlautung Zeichen, füge neue ein, lasse andere weg. Kultur setze sich in Sprache um und Sprache schlage sich in der Schrift nieder. Die Alphabete und ihre Entwicklung sind somit der Seismograph der Menschheitsgeschichte. Büttners erklärtes Ziel war es, „sämtliche Buchstaben nach ihrer natürlichen Verwandschaft" zu beschreiben (Büttner 1779: 9). Er halte Wörter, schrieb er einleitend, für die „Hauptbestimmungszeichen“ der Völker. Es werde sich zeigen, wenn man die alten mit den neuen Sprachen vergleiche, ,daß wenigere Stammvölker als man gemeiniglich glaubt, gänzlich ausgestorben sind“ (Büttner 1771: 1). Im 
ersten seiner zwei Hefte publizierte er dazu drei Tabellen, die die Entwicklung der antiken Alphabete nachvollziehen lassen, und skizzierte damit den Ausgangpunkt. Im zweiten Heft versuchte er sämtliche zeitgenössischen Alphabete in einer phonetisch-systematischen Gesamtschau als Tafel zu präsentieren.

Wie für Gatterer in den Urkunden und eine Ebene tiefer in der Schriftentwicklung hat sich für Büttner die Geschichtsentwicklung in der Alphabetentwicklung eingeschrieben. Und wie Gatterers Übersetzung des Linnaeismus graphicus zur Chronometrie barg auch Büttners Unternehmen einen eminent praktischen, hier ausgesprochen aufklärerischen Sinn. Man müsse beim Vergleich der Sprachen mit der Alphabetsanalyse beginnen und darauf sehen, ob sie auch die Laute der illiteraten Völker umfassten. Derart ließe sich ein Weltalphabet konstruieren, das alle semantisch genutzten menschlichen Laute beinhalte. Statt wie die Römer anderen Völkern ihre Sprache aufzudrängen und damit deren Kultur zu zerstören, ließe es das Weltalphabet zu, illiterate Völker in die Weltöffentlichkeit aufzunehmen und gestatte ihnen, ihre eigene Sprache, Geschichte und Verfassung aufzuzeichnen. Ein Weltalphabet mache es möglich, ihre Gedanken für die Allgemeinheit $\mathrm{zu}$ erfassen, und biete ihnen andererseits, was die Missionierung angehe, die Chance, das Evangelium selbst zu durchdenken und aufgrund seiner Güte anzunehmen, statt dass es diesen Völkern nur aufoktroyiert würde (Büttner 1779: $4 \mathrm{f}$.).

Herausgekommen ist die 48-spaltige Tabelle des Weltalphabets, die in den ersten 47 Spalten die 22 von Büttner konstatierten Alphabete der Welt und ihre maßgeblichen Varianten enthält (Abb. 7). In der 48. Spalte, rechts abgetrennt, befindet sich dann das Weltalphabet, für das Büttner den aus den Alphabeten gewonnenen Phonemen je ein eindeutiges Zeichen aus den lateinischen, griechischen und kyrillischen zugeordnet hatte. Links davon stehen entsprechend die "Aramäischen Alphabete", das chaldäische, hebräische, phönizische, samaritanische, syrische, sowie drei Varianten des arabischen Alphabets, dann das persische und armenische. Beginnend mit den Runen in Spalte 15 hat Büttner die okzidentalen Alphabete eingetragen, nach dem griechischen, lateinischen, neogotischen das kyrillische und koptische. Ab Spalte 24 folgen Silbenschriften, die äthiopische, mongolische, tibetanische, in Spalte 41 die siamesische, in 42 die javanesische, in den Spalten 43-46 japanische Schriften und in Spalte 47 schließlich der Versuch, chinesische Schriftzeichen lautlich zu erfassen. In der Breite ist das Projekt entwicklungsgeschichtlich kartographiert (geographisch und kulturell im Osten beginnend über den Westen 
Tabula $I$.

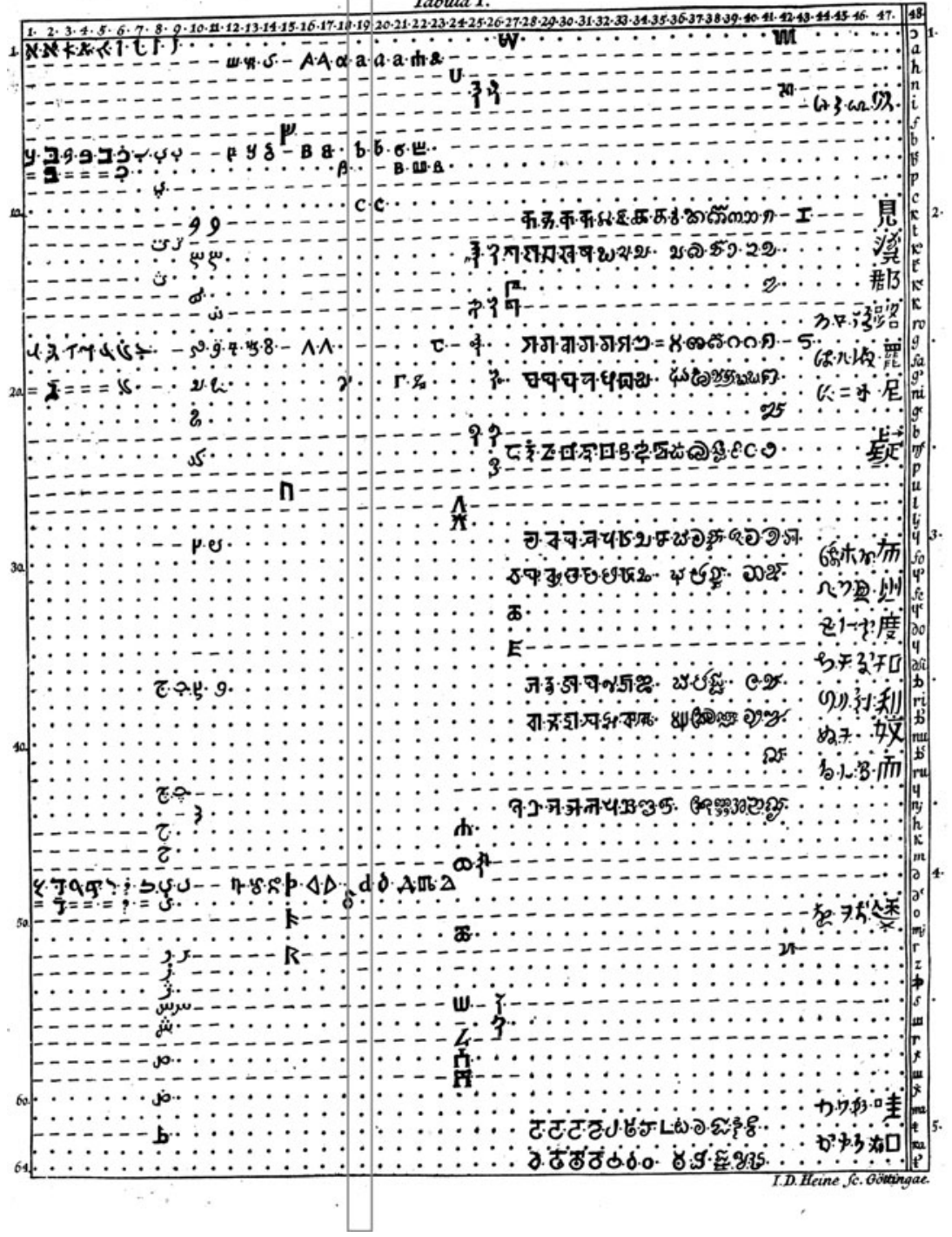

und dann weiter wieder in den Osten), in der Tiefe umfasst es Schrift lautlich und besteht insgesamt aus 360 Zeilen (Büttner 1776: 109 f.).

In der Mitte des 19. Jahrhunderts veröffentlichte der Begründer der deutschen Ägyptologie, Karl Richard Lepsius, sein Allgemeines linguistisches Alphabet, eine Umschrift zur Transkription von Sprache. ${ }^{16}$ Damit wollte er zum einen fremde Völker für die wissenschaftliche Erforschung zugänglich machen, zum anderen den „Völkern, namentlich den heidnischen und noch gänzlich uncivilisierten, eine zweckmässige Schrift [...] geben“, um
Abb. 7: Büttners Weltalphabet. Lateinisches Alphabet zur Orientierung überblendet (Büttner 1776: 109). 
dadurch „die Ausbreitung des christlichen Glaubens und der christlichen Civilisation“ und die „Missionsthätigkeit“ zu erleichtern (Lepsius 1855: 1). Lepsius' Alphabet wurde zum Vorläufer des Internationalen Phonetischen Alphabets, auf das sich inzwischen wiederum die Umsetzung von geschriebener Sprache in Computercodierungen stützt. Konzeptionell gesehen ist der Weg von Büttner über Lepsius zu Google und der digitalen Welt kürzer, als es den Anschein haben mag.

\section{Botanik, Diplomatik, Linguistik, Ethnographie - die Schrift der Natur und die Natur der Schrift}

Die Entwicklung der Klassifikation hin zu Evolutionstheorien ist nach derzeitigem Erkenntnisstand - nicht über Büttner, Gatterer und die Benediktiner gelaufen, auch wenn in der Diplomatik früher als in der Naturgeschichte nolens volens massiv Datierungsfragen erörtert wurden und Büttner in seiner linguistischen Ethnographie mit den Sprachlauten begann, sich um ein inneres Funktionselement von Sprache zu kümmern. Wenn Büttner die Entwicklung der Alphabete und Lautung notierte, verstand auch er die Phonetik als Instrument auf dem Weg zu einer allgemeinen Sprachtranskription und als Mittel, Völkergeschichte zu betreiben. Die lautliche und Alphabetentwicklung selbst und ihre Entwicklungsgesetze hatte er nicht im Sinn. Linnés Fruktationsbuchstaben, Büttners Hauptbestimmungszeichen und Gatterers Schriftengattungen der Völker demonstrieren jedoch, wie selbstverständlich Mitte des 18. Jahrhunderts erwartet wurde, das Wesen der Dinge konkret in den Dingen eingeschrieben zu finden: Natur wie Kultur erschienen nicht chaotisch, weil sie einer eingeschriebenen Sprache folgten und damit Ordnung, Sinn und System zu bilden vermochten. Sie besaßen ein Alphabet und mit ihm und der eingeschriebenen Sprache offenkundig natürliche Kultur und kultivierte Natur.

Legt man Linnés, Gatterers und Büttners Ausbuchstabieren des Alphabets der Natur und Kultur nebeneinander, zeigt sich: Für alle drei sind Natur und Kultur direkt verbunden, und die Berücksichtigung dieser Verbindung erscheint um so mehr methodisch geboten, da es um das empirische Erfassen der jeweiligen Gegenstände geht. Durch die Verknüpfung erweist sich der jeweilige Ansatz als schöpfungs- wie naturgerecht. Die Überzeugung, die jeweiligen Alphabete zu rekonstruieren, legitimierte Forschung nicht zuletzt auch aus praktischen Erwägungen. Ließen sich die 
Alphabete finden, hatte man die innere Ordnung gewonnen, die sich instrumentell zu Forschungs- und Kommunikationswerkzeugen ausbauen ließ und so praktisch den Aufklärungsglauben an die Identität von Vernunft, Nutzen und Ordnung belegte. Bei Linné lässt sich Botanik als umfassendes naturgemäßes Klassifizieren erst betreiben, weil es das Alphabet der Pflanzen gibt. Bei Gatterer ist der Linnaeismus graphicus das Hilfsmittel, um Schriftentwicklung und mit ihr Geschichte zu verifizieren. Bei Büttner steht das Lautalphabet für den Ausgangspunkt einer sich verstehenden, interethnisch kommunizierenden Welt.

Ein viertes kommt zum weltbildlichen, methodischen und praktischen Gebrauch der Alphabete hinzu: Bestand Konsens über die Verknüpfung von Kultur und Natur, so hatte der jeweilige Fachdiskurs notwendig die Entwicklung der Fragen und Vorgehensweisen der anderen Fachdispute zu berücksichtigen, solange und in dem Maße, wie die zwar gegliederte, aber umfassende Res publica litteraria reale und nicht nur normative Bedeutung besaß. In der Perspektive der empirischen Beobachtung einer kulturgemäßen Natur Linnés offerierte die Kultur extern den Gliederungsrahmen. In Gatterers Perspektive der Bestandsaufnahme naturgemäßer Schreibkultur offerierte die Natur intern die Historisierung qua Entwicklungsaspekt der Formen. In der Verschränkung von Natur und Kultur in der Ethnographie Büttners schließlich verschmolzen Laut (Natur) und Völkergeschichte (Kultur) zum weltumspannenden Zeichensystem. Die Ausdifferenzierung von Natur- und Kulturwissenschaften scheint in der Mitte des 18. Jahrhunderts noch kein geradlinig vorgezeichneter Weg gewesen zu sein, sondern ein Stück weit ein gegenseitiges Ausprobieren und Abtasten, bei dem das aus vielen Gründen Passendste Fortsetzung fand, ohne dass die Grundkonzeption dadurch verloren gegangen wäre. Büttners Weltalphabet ist ein Versuch geblieben, aber die Verbindung von Laut, Zeichen und Sprache ist noch für die heutige Linguistik zentral, und Gatterers Analyse der Buchstabenformen-Entwicklung wurde vor einigen Jahren von der computergestützten Paläographie wiederentdeckt (Bromm 1995: 155, Burkardt 1997: 22).

Die Idee des Alphabets als Metapher, Metonymie, Modell und Methodenschema hielt die gelehrte Welt zusammen, umfasste dabei Wissenschaftsvorstellungen, -sprache und -praxis und ließ es zu, im Fortgang der wissenschaftlichen Praxis mehr und mehr zwischen Metapher, Metonymie und Modell zu selektieren. Gatterer, Linné und Büttner motivierten die Möglichkeiten, die das 
Alphabet der Natur und das Alphabet der Kultur augenscheinlich bot, die empirische Welt in Fachsprachen zu übersetzen und diese wissenschaftspraktisch, aber auch kulturell zu nutzen.

\section{Danksagung}

Ich danke dem Max-Planck-Institut für Wissenschaftsgeschichte Berlin und der Gerda-Henkel-Stiftung für die Förderung der Forschungsarbeiten, auf denen dieser Artikel beruht.

Open Access This article is distributed under the terms of the Creative Commons Attribution Noncommercial License which permits any noncommercial use, distribution, and reproduction in any medium, provided the original author(s) and source are credited.

\section{Anmerkungen}

1 Zu Johann Christoph Gatterer und der Geschichtsschreibung im 18. Jahrhundert bereite ich derzeit eine Monographie für den Druck vor.

2 Müller-Wille 1999, Mayr 1982, Lefèvre 1984, Larson 1971, 1994.

3 Vgl. zur „Ökonomie der Natur“ Morgenthaler 2000: 95-105.

4 Müller-Wille 2005, Weigel u. a. 2005, Lefèvre 1984: 228-232, Pörksen 1986: $72 \mathrm{f}$.

5 Im lateinischen Original heißt es: „FRUCTIFICATIO [...] Vegetabilium pars temporaria, Generationi dicata, antiquum terminans, novum incipiens; hujus Partes VII. numerantur."

6 Blanke 1991, Muhlack 1991, Reill 1975, 1980, Friederichs 1977, Hammerstein 1972.

7 Gatterer 1765: 81-143, 1798: 39-53, 1806: 34-45; vgl. Rödel 1982.

8 Vgl. Bresslau 1968, Foerster/Frenz 2004, Tropper 1994, Vogtherr 2008.

9 Vgl. [Toustain/Tassin] 1750-1765, Bd. 1: 8 f., 127 f., Bd. 2: 348 f. (Übersetzung [Adelung] 1759-1769, Bd. 1: 60 f., Bd. 3: 40 f.), Massiczek 1939.

10 In den Elementa konzipierte er „Currentschriften, gemeine Schriften“ als viertes „Regnum privatum“ (Gatterer 1765: 82); später ordnete er sie in das „Urkundenschrift-Gebiet“" ein (Gatterer 1798: 38).

11 Vgl. [Gatterer] 1786: 1945; zu Schöpflin vgl. Voss 1979. Die Datierung spreche für Gatterers Methode, urteilte Tropper (1994: 135). Zur Peutinger Karte vgl. Weber 1976; zur Paläographie in ihrer entwickelten Form im Detail vgl. Foerster/Frenz 2004.

12 Vgl. Pörksen 1986: 72.

13 Vgl. zu Büttner: Urban 1991, 2001, Plischke 1931, Schlichtegroll 1802, Nawa 2005.

14 Zur Geschichte der Alphabetforschung vgl. Drucker 1995, Haarmann 1990, 2002; zum „,natürlichen Alphabet“ und zur Phonetik vgl. Gessinger 1994: 633-719.

15 Büttner 1779: 6 f.; zur Sprachwissenschaft im 18. Jahrhundert vgl. Gardt 1999, Gessinger 1994, Semsch 1999, Tintemann/Trabant 2004.

16 Lepsius 1855, vgl. Drucker 1995: 257, Haarmann 1990: 299, Gessinger 1994: 640-647. 


\section{Literatur}

[Adelung, Johann Christoph], 1759-1769. Neues Lehrgebäude der Diplomatik, welches in Frankreich von einigen Benedictinern von der Congregation des heil. Mauri ausgefertiget worden. 9 Bde. Unter Mitarbeit von Hermann Ernst Rumpel, übersetzt von Anton Rudolph (ab Bd. 4). Erfurt: Johann Friedrich Weber.

Blanke, Horst Walter, 1991. Historiographiegeschichte als Historik. Stuttgart: Frommann-Holzboog.

Blanke, Horst Walter/Fleischer, Dirk, 1990. Theoretiker der deutschen Aufklärungshistorik. 2 Bde. Stuttgart: Frommann Holzboog.

Breger, Claudia, 1995. Heinrich Moritz Gottlieb Grellmann. Überlegungen zu Entstehung und Funktion rassistischer Deutungsmuster im Diskurs der Aufklärung. In: Barbara Danckwortt, Hg., Historische Rassismusforschung. Ideologien - Täter - Opfer. Berlin: Argument, 34-69.

Bresslau, Harry, 1968. Handbuch der Urkundenlehre für Deutschland und Italien. 2 Bde. Berlin: De Gruyter [2. Aufl.].

Bromm, Gudrun, 1995. Die Entwicklung der Großbuchstaben im Kontext hochmittelalterlicher Papsturkunden. Marburg: Institut für Historische Hilfswissenschaften.

Burkardt, Johannes, 1997. Die Historischen Hilfswissenschaften in Marburg (17.-19. Jahrhundert). Marburg: Institut für Historische Hilfswissenschaften.

Büttner, Christian Wilhelm, 1771. Vergleichungs-Tafeln der Schriftarten verschiedener vergangener und gegenwärtiger Zeiten. Stück 1. Göttingen: Dieterich.

Büttner, Christian Wilhelm, 1776. Brevis expositio alphabetorum omnium populorum et affinitatum quibus illa inter se coniuncta sunt. Novi Commentarii Societatis Regiae Scientiarum Gottingensis, 7, 106-126.

Büttner, Christian Wilhelm, 1779. Vergleichungs-Tafeln der Schriftarten verschiedener vergangener und gegenwärtiger Zeiten. Stück 2. Göttingen: Dieterich.

Drucker, Johanna, 1995. The Alphabetic Labyrinth. The Letters in History and Imagination. London: Thames \& Hudson.

Feuerstein-Herz, Petra, 2007. „Die große Kette der Wesen“. Ordnungen in der Naturgeschichte der Frühen Neuzeit. Wolfenbüttel: Herzog August Bibliothek.

Foerster, Hans/Frenz, Thomas, 2004. Abriß der lateinischen Paläographie. Stuttgart: Anton Hiersemann Verlag [2. Aufl.].

Friederichs, Heinz F., 1977. Johann Christoph Gatterer und seine Bedeutung für die wissenschaftliche Genealogie. Genealogisches Jahrbuch, 16/17, 41-47.

Gardt, Andreas, 1999. Geschichte der Sprachwissenschaft in Deutschland. Berlin: De Gruyter.

Gatterer, Johann Christoph, 1765. Elementa artis diplomaticae universalis. Göttingen: Vandenhoeck \& Ruprecht.

Gatterer, Johann Christoph, 1767. Nouveau Traité de diplomatique (Rezension). Allgemeine historische Bibliothek von Mitgliedern des königlichen Instituts des historischen Wissenschaften zu Göttingen, 1, 161-212.

[Gatterer, Johann Christoph], 1786, o. T. (Bericht über den Akademievortrag vom 18. November 1786). Göttingische Anzeigen von Gelehrten Sachen, 194, 1945-1952.

Gatterer, Johann Christoph, 1787. Commentatio diplomatica de methodo aetatis codicum manuscriptorum definiendae. Commentationes Societatis Regiae Scientiarum Gottingensis. Hist. U. phil. Kl., 8, 85-121.

Gatterer, Johann Christoph, 1798. Abriß der Diplomatik. Göttingen: Vandenhoeck \& Ruprecht.

Gatterer, Johann Christoph, 1799. Praktische Diplomatik. Göttingen: Vandenhoeck \& Ruprecht.

Gatterer, Johann Christoph, 1806. Epitome artis diplomaticae. Salzburg: Caspar Zaunrith [2. Aufl.].

Gessinger, Joachim, 1994. Auge E Ohr. Studien zur Erforschung der Sprache am Menschen 1700-1850. Berlin: De Gruyter. 
Grellmann, Heinrich Moriz Gottlieb, 1787. Historischer Versuch über die Zigeuner betreffend die Lebensart und Verfassung, Sitten und Schicksale dieses Volks seit seiner Erscheinung in Europa und dessen Ursprung. Göttingen: Dieterich [2. Aufl.].

Haarmann, Harald, 1990. Universalgeschichte der Schrift. Frankfurt a. M.: Campus. Haarmann, Harald, 2002. Geschichte der Schrift. München: Beck.

Hammerstein, Notker, 1972. Jus und Historie. Ein Beitrag zur Geschichte des historischen Denkens an deutschen Universitäten im späten 17. und 18. Jahrhundert. Göttingen: Vandenhoeck \& Ruprecht.

Hund, Wulf Dietmar, Hg., 1996. Zigeuner. Geschichte und Struktur einer rassistischen Konstruktion. Duisburg: Unrast.

Larson, James L., 1971. Reason and Experience. The Representation of Natural Order in the Work of Carl von Linné. Berkeley: University of California Press.

Larson, James L., 1994. Interpreting Nature. The Science of Living From Linnaeus to Kant. Baltimore: Johns Hopkins University Press.

Lefèvre, Wolfgang, 1984. Die Entstehung der biologischen Evolutionstheorie. Wien: Ullstein.

Lefèvre, Wolfgang, 2003. Changing Contexts of Classification. Cuvier's Transformation of Biological Classification. In: Ursula Klein, Hg., Spaces of Classification. Berlin: Max-Planck-Institut für Wissenschaftsgeschichte (Preprint 240), 1-13.

Lepsius, Karl Richard, 1855. Das Allgemeine linguistische Alphabet. Grundsätze der Übertragung fremder Schriftsysteme und bisher noch ungeschriebener Sprachen in europäische Buchstaben. Berlin: Wilhelm Hertz.

Linné, Carl von, 1737. Genera plantarum eorumque characteres naturales. Secundum numerum, figuram, situm, proportionem omnium fructificationis partium. Leiden: Wishoff.

Linné, Carl von, 1749/1787. Oeconomia naturae. In: Ders., Caroli Linnaei Amoenitates academicae, Bd. 2. Erlangen: J. Jakob Palm, 2-58 [3. Aufl.].

Linné, Carl von, 1751. Philosophia botanica in qua explicantur fundamenta botanica cum definitionibus partium, exemplis terminorum, observationibus rariorum. Stockholm: Kiesewetter.

Linné, Carl von, 1775. Gattungen der Pflanzen und ihre natürliche Merkmale nach der Anzahl, Gestalt, Lage und Verhältniß aller Blumentheile. Nach der sechsten Ausgabe und der ersten und zweyten Mantisse übersetzt von Johann Jakob Planer. Bd. 1. Gotha: Ettinger.

Lindman, C.A.M., 1909. Carl von Linné als botanischer Forscher und Schriftsteller. In: Carl von Linnés Bedeutung als Naturforscher und Arzt. Hg. von der Kg. Schwedischen Akademie der Wissenschaften. Jena: Fischer.

Massiczek, Albert, 1939. Die ideengeschichtlichen Grundlagen des Nouveau Traité de Diplomatique. Wien: Dissertation.

Mayr, Ernst, 1982. The Growth of Biological Thought. Cambridge: Cambridge University Press.

Morgenthaler, Erwin, 2000. Von der Ökonomie der Natur zur Ökologie. Die Entwicklung ökologischen Denkens und seiner sprachlichen Ausdrucksformen. Berlin: Schmidt.

Muhlack, Ulrich, 1991. Geschichtswissenschaft im Humanismus und in der Aufklärung. Die Vorgeschichte des Historismus. München: Beck.

Müller-Wille, Staffan, 1999. Botanik und weltweiter Handel. Zur Begründung eines Natürlichen Systems der Pflanzen durch Carl von Linné (1707-1778). Berlin: Verlag für Wissenschaft und Bildung.

Müller-Wille, Staffan, 2005. Konstellation, Serie, Formation. Genealogische Denkfiguren bei Harvey, Linnnaeus und Darwin. In: Sigrid Weigel, Ohad Parnes, Ulrike Vedder und Stefan Willer, Hg., Generation. Zur Genealogie des Konzepts - Konzepte von Genealogie. München: Fink, 215-234.

Nawa, Christine, 2005. Sammeln für die Wissenschaft. Das Academische Museum Göttingen (1773-1849). Göttingen: Magisterarbeit an der philosophischen Fakultät.

Pernkopf, Elisabeth, 2004. Alphabetisierte Natur? Sprachformen in den Naturwissenschaften. In: Reinhold Esterbauer, Elisabeth Pernkopf und Mario 
Schönhart, Hg., Spiel mit der Wirklichkeit. Zum Erfahrungsbegriff in den Naturwissenschaften. Würzburg: Königshausen \& Neumann, 81-94.

Plischke, Hans, 1931. Die Ethnographische Sammlung der Universität Göttingen. Ihre Geschichte und ihre Bedeutung. Göttingen: Vandenhoeck \& Ruprecht.

Pörksen, Uwe, 1986. Deutsche Naturwissenschaftssprachen. Tübingen: Narr.

Rassem, Mohammed/Stagl, Justin, Hg., 1980. Statistik und Staatsbeschreibung in der Neuzeit vornehmlich im 16.-18. Jahrhundert. Paderborn: Schöningh.

Reill, Peter Hanns, 1975. The German Enlightenment and the Rise of Historicism. Berkeley: University of California Press.

Reill, Peter Hanns, 1980. Johann Christoph Gatterer. In: Hans Ulrich Wehler, Deutsche Historiker, Bd. 6. Göttingen: Vandenhoeck \& Ruprecht, 7-22.

Rödel, Volker, 1982. Johann Christoph Gatterers „Linnaeismus graphicus“. Klasssifikation und Universalismus im 18. Jahrhundert. Medizinhistorisches Journal, 17, 215-238.

Schlichtegroll, Friedrich, Hg., 1802/2004. Artikel: Christian Wilhelm Büttner. Nekrolog der Teutschen für das 19. Jahrhundert. Bd. 1. In: Deutsches Biographisches Archiv. München: Saur, Mikrofiche I 163, 188-216.

Schulz Michael/Kuhn Hans-Jürg, 2001. Die Blumenbachsche Schädelsammlung. In: Dietrich Hoffmann und Kathrin Maack-Rheinländer, Hg., „Ganz für das Studium angelegt". Die Museen, Sammlungen und Gärten der Universität Göttingen. Göttingen: Wallstein, 169-174.

Semsch, Klaus, 1999. Abstand von der Rhetorik. Strukturen und Funktionen ästhetischer Distanznahme von der "Ars Rhetorica" bei den französischen Enzyklopädisten. Hamburg: Meiner.

Tintemann, Ute/Trabant, Jürgen, Hg., 2004. Sprache und Sprachen in Berlin um 1800. Hannover: Wehrhahn.

[Toustain, Charles François/Tassin, René Prosper], 1750-1765. Nouveau Traité de diplomatique, où l'on examine les fondemens de cet art par deux religieux Bénédictins de la Congrégation de S. Maur. 6 Bde. Paris: Guillaume Desprez.

Tropper, Peter G., 1994. Urkundenlehre in Österreich vom frühen 18. Jahrhundert bis zur Errichtung der "Schule für Österreichische Geschichtsforschung" 1854. Graz: Akad. Druck- und Verlagsanstalt.

Urban, Manfred, 1991. Die völkerkundliche Sammlung der Universität Göttingen. Georgia Augusta - Nachrichten aus der Universität Göttingen, 55, 17-29.

Urban, Manfred, 2001. Die Völkerkundliche Sammlung. Eine im Zeitalter der Aufklärung wurzelnde ethologische Sammlung ihre Entstehung und weitere Entwicklung. In: Dietrich Hoffmann und Kathrin Maack-Rheinländer, Hg., „Ganz für das Studium angelegt". Die Museen, Sammlungen und Gärten der Universität Göttingen. Göttingen: Wallstein, 91-98.

Vogtherr, Thomas, 2008. Urkundenlehre. Hannover: Hahnsche Buchhandlung.

Voss, Jürgen, 1979. Universität, Geschichtswissenschaft und Diplomatie im Zeitalter der Aufklärung: Johann Daniel Schöpflin (1694-1771). München: Fink.

Weber, Ekkehard, 1976. Tabula Peutingeriana. Graz: Akad. Druck- und Verlags-Anstalt. Weigel, Sigrid/Parnes, Ohad/Vedder, Ulrike/Willer, Stefan, Hg., 2005. Generation. Zur Genealogie des Konzepts - Konzepte von Genealogie. München: Fink.

\section{Martin Gierl}

Seminar für Mittlere und

Neuere Geschichte

Universität Göttingen

Platz der Göttinger Sieben 5

D-37073 Göttingen, Deutschland

E-Mail: mgierl1@gwdg.de 